\title{
ON A NUMBER OF SOLUTIONS IN PROBLEMS WITH SPECTRAL PARAMETER FOR EQUATIONS WITH DISCONTINUOUS OPERATORS
}

\author{
D.K. POTAPOV
}

\begin{abstract}
In a real reflexive Banach space we consider a problem on existence of solutions to a problem with a spectral parameter for equations with discontinuous operators. By the variational approach we obtain theorems on the number of the solutions to the considered problems. As an application, we consider main boundary value problems for elliptic equations with a spectral parameter and discontinuous nonlinearities.
\end{abstract}

Keywords: spectral parameter, discontinuous operator, variational method, number of solutions.

Mathematics Subject Classification: 47J10, 47J30, 35P30, 35J60, 35J20.

\section{Introduction. Formulation OF PROBlEM}

General formulation of the eigenvalue problems for nonlinear equations was given in work [1]. In works [1], 2], nonlinear problems with a spectral parameter were studied by topological methods, in works [3], 4], they were treated in semi-ordered spaces, and in works [5], [6], a variational method was employed. In all cited works the structure of the set of eigenvalues for operator equation was studied for continuous mappings. In the present work we consider nonlinear spectral problems in a general operator formulation without assuming continuity for the operator. In what follows we shall make use of the following definitions.

Let $E$ be a real reflexive Banach space, $E^{*}$ be the adjoint space for $E$. By $(z, x)$ we denote the action of a functional $z \in E^{*}$ on an element $x \in E$.

Definition 1. A linear operator $A: E \rightarrow E^{*}$ is called self-adjoint if $(A x, h)=(A h, x)$ for all $x, h \in E$.

Definition 2. A mapping $T: E \rightarrow E^{*}$ is called compact on $E$ if it maps bounded sets in $E$ into precompact ones in $E^{*}$.

Definition 3. A mapping $T: E \rightarrow E^{*}$ is called monotone on $E$ if $(T x-T y, x-y) \geq 0$ for all $x, y \in E$. A mapping $T: E \rightarrow E^{*}$ is called anti-monotone if the mapping $-T$ is monotone.

Definition 4. A mapping $T: E \rightarrow E^{*}$ is called bounded on $E$ if there exists a constant $M>0$ such that $\|T x\| \leqslant M$ for all $x \in E$.

In the present work we consider the equation

$$
A u=\lambda T u
$$

with a parameter $\lambda>0$. Here $A$ is a linear self-adjoint operator acting from $E$ into $E^{*}$, $T: E \rightarrow E^{*}$ is a discontinuous compact or anti-monotone mapping bounded on $E$.

D.K. Potapov, On A NUMBer of SOlUtions in PROBlems With SPECTRAL PARAMETER FOR EQUATIONS WITH DISCONTINUOUS OPERATORS.

(C) Potapov D.K. 2013.

Submitted February 4, 2012. 
Works [7], 8] were devoted to the existence of a ray of positive eigenvalues for such equations with discontinuous operators. The theorems on existence of non-zero solutions to equation (1) were proven for sufficiently large $\lambda$. In works [9], [10], the estimates for the value of the bifurcation parameter and for the norms of the operator in spectral problems for equations with discontinuous operators of the form (1) were established. The existence of zero solution of equation (1) for each $\lambda$ is ensured by the assumption $T(0)=0$. In the present work we consider the question on the number of solutions to equation (1).

\section{General Results}

As earlier [7]-[10], equation (1) is studied by the variational method. To ensure the applicability of this approach to equation (1), we suppose in addition that the operator $T$ is quasipotentional.

Definition 5. A mapping $T: E \rightarrow E^{*}$ is called quasipotentional if there exists a functional $f: E \rightarrow \mathbf{R}$ obeying the identity $f(x+h)-f(x)=\int_{0}^{1}(T(x+t h), h) d t$ for all $x, h \in E$ (the integral as treated as the Lebesgue one). At that, $f$ is called a quasipotential of the operator $T$.

To equation (1) we associate the functional $f^{\lambda}(u)=\frac{1}{2}(A u, u)-\lambda f(u)$, where $f$ is the quasipotential of the operator $T$. In work [11], there were given the sufficient conditions (restrictions for the points of discontinuity of the operator $F_{\lambda} u=A u-\lambda T u$ ) such that the minima of the functional $f^{\lambda}$ are solutions to equation (1). Namely, one should assume that the points of discontinuity of the operator $F_{\lambda}$ are regular.

Definition 6. An element $x \in E$ is called a point of discontinuity of an operator $T: E \rightarrow E^{*}$ if there exists $h \in E$ such that either $\lim _{t \rightarrow 0}(T(x+t h), h)$ does not exist or $\lim _{t \rightarrow 0}(T(x+t h), h) \neq$ $(T x, h)$.

Definition 7. An element $x \in E$ is called a regular point of an operator $T: E \rightarrow E^{*}$ if $\varlimsup_{t \rightarrow+0}(T(x+t h), h)<0$ for some $h \in E$.

According to the results of works [7], 8], the following theorems hold true.

Theorem 1. Suppose

1) $A$ is a linear self-adjoint operator acting from a real reflexive Banach space $E$ into the adjoint space $E^{*}$; the space $E$ is represented as the direct sum of closed subspaces $E_{1}$ and $E_{2}$, $E_{1}=\operatorname{ker} A$, and there exists a constant $\alpha>0$ such that $(A u, u) \geq \alpha\|u\|^{2}$ for all $u \in E_{2}$;

2) a mapping $T$ is compact or anti-monotone, quasipotentional (with a quasipotential $f$ ), and bounded on $E, f(0)=0$ and for some $u_{0} \in E$ the inequality $f\left(u_{0}\right)>0$ holds; if $E_{1} \neq\{0\}$, it is assumed in addition that $\lim _{u \in E_{1},\|u\| \rightarrow+\infty} f(u)=-\infty$;

$3)$ if the mapping $T$ is compact, it is assumed in addition that $\lim _{t \rightarrow+0}(T(u+t h)-T u, h) \geq 0$ for all $u, h \in E$;

4) if the mapping $T$ is anti-monotone, it is assumed in addition that each point of discontinuity for the operator $T$ is regular for $F_{\lambda} u=A u-\lambda T u$ when $\lambda>\lambda_{0}>0\left(\lambda_{0}\right.$ is a quantity starting from which the eigenvalue problem is solvable).

Then for each $\lambda>\lambda_{0}$ equation (1) has at least one non-zero solution.

Note that if we additionally suppose that $T(0)=0$ under the conditions of Theorem 1 , then for the compact mapping $T$ equation (1) has at least two solutions (zero and non-zero) for each $\lambda>\lambda_{0}$, while if the mapping $T$ is anti-monotone, equation (1) has only trivial solution, since in this case non-zero solutions are impossible. 
A central notion of modern variational theory is the Palais-Smale condition ((PS)-condition) and its basis is the deformation lemma. Basing on the notion of the Clarke generalized gradient for locally Lipshitz functions, (PS)-condition and the deformation lemma were modified by K.C. Chang [12].

Definition 8. A function $f: E \rightarrow \mathbf{R}$ is called locally Lipshitz if for each $x \in E$ there exists a neighborhood $U$ of the point $x$ and a constant $L>0$ such that $|f(u)-f(v)| \leqslant L\|u-v\|$ for all $u, v \in U$.

Definition 9. A generalized derivative in the direction $l$ of a locally Lipshitz function $f$ at a point $x$ is $f^{\circ}(x, l)=\varlimsup_{z \rightarrow x, t \rightarrow+0} \frac{f(z+t l)-f(z)}{t}$, and generalized derivative of $f$ at the point $x$ is the set $\partial f(x)=\left\{y \in E^{*}: f^{\circ}(x, l) \geq(y, l) \quad \forall l \in E\right\}$.

Definition 10. A locally Lipshitz function $f: E \rightarrow \mathbf{R}$ satisfies (PS)-condition if each sequence $\left(x_{n}\right) \subset E$ such that the set $\left(f\left(x_{n}\right)\right)$ is bounded and $m\left(x_{n}\right)=\inf _{x^{*} \in \partial f\left(x_{n}\right)}\left\|x^{*}\right\| \rightarrow 0$ as $n \rightarrow \infty$ contains a convergent sequence, where $\partial f(x)$ is the Clarke generalized gradient for $f$ at the point $x$.

The main result of the present work is the following theorem.

Theorem 2. Let $E$ be a real Hilbert space densely and compactly embedded into a real reflexive Banach space $E_{3}, A: E \rightarrow E$ be a linear self-adjoint bounded operator, zero be an isolated point in its spectrum the kernel and negative subspace of the operator $A$ be finite-dimensional, and Conditions 2)-3) of Theorem 1 hold for a mapping $T: E_{3} \rightarrow E_{3}^{*}, T(0)=0$. Then there exists $\lambda_{*}>0$ such that for each $\lambda>\lambda_{*}$ equation (1) has at least three solutions.

Proof. It is known [13] that if $E$ is a Hilbert space, Condition 1) of Theorem 1 holds true once zero is an isolated point in the spectrum of a non-negative operator $A$. In this case there exists a constant $\alpha>0$ such that $(A u, u) \geq \alpha\|u\|^{2} \forall u \in E_{2}\left(E_{1}=\operatorname{ker} A, E_{2}=E_{1}^{\perp}\right)$. This fact, the conditions of the theorem, and Theorem 1 imply the existence of $\lambda_{0}>0$ such that for each $\lambda>\lambda_{0}$ equation (1) has at least one non-zero solution, i.e., for some $\lambda_{*}>0$ for each $\lambda>\lambda_{*}$ there exists an element $u_{\lambda} \in E, u_{\lambda} \neq 0$ such that $f^{\lambda}\left(u_{\lambda}\right)=\inf _{v \in E} f^{\lambda}(v)<0$, as it follows from Theorem 2 in work [7]. Let us show that for $\lambda>\lambda_{*}$ equation (1) has at least one more non-trivial solution $v_{\lambda}$ that can be found by mountain pass theorem [12] if $f^{\lambda}\left(v_{\lambda}\right)>0$. To satisfy the conditions of mountain pass theorem [12], it is sufficient to show that the function $f^{\lambda}$ satisfies (PS)-condition for each $\lambda>0$. In order to do this, it is enough to prove that the function $f^{\lambda}$ is locally Lipshitz on $E$ (all other assumptions of Theorem 4.5 in [12] are identical to ones of this theorem). Indeed, this is true, since $A$ is a linear bounded operator and the function $f$ satisfies Lipshitz condition on $E_{3}$, since for arbitrary $u, v \in E_{3}$ we have

$$
\begin{gathered}
|f(u)-f(v)|=\left|\int_{0}^{1}(T(v+t(u-v)), u-v) d t\right| \leqslant \\
\int_{0}^{1}|(T(v+t(u-v)), u-v)| d t \leqslant M\|u-v\|_{E_{3}},
\end{gathered}
$$

because the mapping $T$ is bounded on $E_{3}, M>0$ is a constant in the inequality $\|T x\| \leqslant M \forall x \in$ $E_{3}$. Hence, by Theorem 4.5 in [12], the functional $f^{\lambda}$ satisfies (PS)-condition for each $\lambda>0$. Therefore, the functional $f^{\lambda}$ satisfies the conditions of mountain pass theorem [12] and has a critical point $v_{\lambda} \in E$ (being a solution to equation (1p) such that $f^{\lambda}\left(v_{\lambda}\right)=\inf _{\gamma \in \Gamma} \sup _{t \in[0,1]} f^{\lambda}(\gamma(t)) \geq$ $\max \left\{f^{\lambda}(0), f^{\lambda}\left(u_{\lambda}\right)\right\}=0\left(\right.$ since $\left.f^{\lambda}(0)=0, f^{\lambda}\left(u_{\lambda}\right)<0\right)$, where $\Gamma=\{\gamma \in \mathbf{C}([0,1], E): \gamma(0)=$ 
$\left.0, \gamma(1)=u_{\lambda}\right\}$. Moreover, by analogy with [7], [14] we can show that $f^{\lambda}(u)>\varepsilon>0$ for $\|u\|=r>0$ and $\left\|u_{\lambda}\right\|>r$. Therefore, there exists $v_{\lambda} \in E$ such that $f^{\lambda}\left(v_{\lambda}\right)>0$. Hence, for each $\lambda>\lambda_{*}$ there exists the second non-trivial solution $v_{\lambda}$, and for each $\lambda>\lambda_{*}$ equation (1) has at least three solutions (zero, $u_{\lambda} \neq 0, v_{\lambda} \neq 0$ ). We observe that solutions $u_{\lambda}$ and $v_{\lambda}$ do not coincide, since $f^{\lambda}\left(u_{\lambda}\right)<0$ and $f^{\lambda}\left(v_{\lambda}\right)>0$. The proof is complete.

\section{Applications}

As an application of the obtained results, we consider the question on the existence of solutions to the problem

$$
\begin{gathered}
L u(x) \equiv-\sum_{i, j=1}^{n}\left(a_{i j}(x) u_{x_{i}}\right)_{x_{j}}+c(x) u(x)=\lambda g(x, u(x)), \quad x \in \Omega, \\
\left.B u\right|_{\Gamma}=0,
\end{gathered}
$$

where $\lambda$ is a positive parameter. Here $L$ is a uniformly elliptic formally self-adjoint differential operator in a bounded domain $\Omega \subset \mathbf{R}^{n}$ with the boundary $\Gamma$ of class $\mathbf{C}_{2, \alpha}(0<\alpha \leqslant 1)$ and with coefficients $a_{i j} \in \mathbf{C}_{1, \alpha}(\bar{\Omega}), c \in \mathbf{C}_{0, \alpha}(\bar{\Omega})$. The function $g: \Omega \times \mathbf{R} \rightarrow \mathbf{R}$ is superpositionally measurable [15] and for almost each $x \in \Omega$ the section $g(x, \cdot)$ has only jump discontinuities on $\mathbf{R}$, $g(x, u) \in\left[g_{-}(x, u), g_{+}(x, u)\right] \forall u \in \mathbf{R}, g_{-}(x, u)=\varliminf_{\eta \rightarrow u} g(x, \eta), g_{+}(x, u)=\varlimsup_{\eta \rightarrow u} g(x, \eta)$. Boundary condition (3) is either the Dirichlet one $\left.u(x)\right|_{\Gamma}=0$, or the Neumann one $\left.\frac{\partial u}{\partial \mathbf{n}_{L}}(x)\right|_{\Gamma}=0$ with the conormal derivative $\frac{\partial u}{\partial \mathbf{n}_{L}}(x) \equiv \sum_{i, j=1}^{n} a_{i j}(x) u_{x_{i}} \cos \left(\mathbf{n}, x_{j}\right)$, where $\mathbf{n}$ is the outward normal to $\Gamma$ and $\cos \left(\mathbf{n}, x_{j}\right)$ are direction cosines of the normal $\mathbf{n}$, or the Robin condition $\frac{\partial u}{\partial \mathbf{n}_{L}}(x)+\left.\sigma(x) u(x)\right|_{\Gamma}=0$, where the function $\sigma \in \mathbf{C}_{1, \alpha}(\Gamma)$ is non-negative and does not identically vanish on $\Gamma$.

Subject to the type of boundary condition (3), we introduce a space $X$. Let $X=\mathbf{H}_{\circ}^{1}(\Omega)$ if $(3)$ is the Dirichlet condition, and $X=\mathbf{H}^{1}(\Omega)$ if $(3)$ is the Neumann or Robin condition. To boundary value problem (2)-(3) we associate a functional $J^{\lambda}$ defined on $X$ as follows: $J^{\lambda}(u)=J_{1}(u)-\lambda J_{2}(u)$, where

$$
J_{1}(u)=\frac{1}{2} \sum_{i, j=1}^{n} \int_{\Omega} a_{i j}(x) u_{x_{i}} u_{x_{j}} d x+\frac{1}{2} \int_{\Omega} c(x) u^{2}(x) d x
$$

in the case of the Dirichlet or Neumann condition, and

$$
J_{1}(u)=\frac{1}{2} \sum_{i, j=1}^{n} \int_{\Omega} a_{i j}(x) u_{x_{i}} u_{x_{j}} d x+\frac{1}{2} \int_{\Omega} c(x) u^{2}(x) d x+\frac{1}{2} \int_{\Gamma} \sigma(s) u^{2}(s) d s
$$

in the case of the Robin condition;

$$
J_{2}(u)=\int_{\Omega} d x \int_{0}^{u(x)} g(x, s) d s .
$$

Definition 11. Let $f: \mathbf{R} \rightarrow \mathbf{R}$. We call $u \in \mathbf{R}$ a jumping discontinuity of the function $f$ if $f(u-)<f(u+)$, where $f(u \pm)=\lim _{s \rightarrow u \pm} f(s)$.

Definition 12. A strong solution to problem (2)-(3) is a function $u \in \mathbf{W}_{r}^{2}(\Omega), r>1$, satisfying equation (2) for almost each $x \in \Omega$ and such that the trace $B u(x)$ on $\Gamma$ vanishes.

Definition 13. A semi-regular solution to problem (2)-(3) is its strong solution u whose values $u(x)$ for almost each $x \in \Omega$ are points of continuity of the function $g(x, \cdot)$. 
In work [12], Clarke's variational calculus was applied for locally Lipshitz functions in proving the existence of strong solutions of the Dirichlet problem for second order elliptic equations with discontinuous nonlinearity and a variational approach for elliptic boundary value problems with discontinuous nonlinearities was developed. Semi-regular solutions were not considered in [12]. In works [7], 8], there were obtained sufficient conditions for the existence of a nontrivial semi-regular solution to problem (2)-(3).

The following theorems hold true.

Theorem 3. Assume

1) $J_{1}(u) \geq 0 \quad \forall u \in X$;

2) for almost each $x \in \Omega$ the function $g(x, \cdot)$ has only jumping discontinuities, $g(x, 0)=0$, and $|g(x, u)| \leqslant a(x) \forall u \in \mathbf{R}$, where $a \in \mathbf{L}_{q}(\Omega), q>\frac{2 n}{n+2}$, is fixed;

3) there exists $u_{0} \in X$ such that $J_{2}\left(u_{0}\right)>0$;

4) if the space $N(L)$ of solutions to the problem

$$
\left\{\begin{array}{l}
L u=0 \\
\left.B u\right|_{\Gamma}=0
\end{array}\right.
$$

is non-zero (the resonance case), we assume in addition that $\lim _{u \in N(L),\|u\| \rightarrow+\infty} J_{2}(u)=-\infty$.

Then there exists $\lambda_{*}>0$ such that for each $\lambda>\lambda_{*}$ problem (2)-(3) has at least three strong solutions, and at least one of non-zero solutions is semi-regular.

Theorem 4. Suppose assumptions 1), 3), 4) of Theorem 3 and $\left.1^{\prime}\right)$ for almost each $x \in \Omega$ the function $g(x, \cdot)$ is non-increasing on $\mathbf{R}$ and for some $a \in \mathbf{L}_{\frac{2 n}{n+2}}(\Omega)$ the inequality $|g(x, u)| \leqslant a(x) \forall u \in \mathbf{R}$ holds true;

2) for almost each $x \in \Omega$ the points of discontinuity of the function $g(x, \cdot)$ are located on the planes $u=u_{i}, i \in I$ ( $I$ is at most countable), and if $g\left(x, u_{i}-\right)>g\left(x, u_{i}+\right)$, then $g\left(x, u_{i}-\right) g\left(x, u_{i}+\right)>0$ for each $i \in I$.

Then there exists $\lambda_{*}>0$ such that for each $\lambda>\lambda_{*}$ problem (2)-(3) has at least one non-zero semi-regular solution.

Proof of Theorems 3, 4. A main condition ensuring the existence of a nontrivial solution to problem (2)-(3) is Condition 3) of Theorem 3. In works [7], [8], it was shown that under the conditions of Theorems 3, 4 there exists $\lambda_{0}>0$ such that for each $\lambda>\lambda_{0} \inf _{v \in X} J^{\lambda}(v)<0$ and there exists $u_{\lambda} \in X$ obeying $J^{\lambda}\left(u_{\lambda}\right)=\inf _{v \in X} J^{\lambda}(v)$ and each such $u_{\lambda}$ is a non-zero semi-regular solution to problem (2)-(3). Thus, there exists $\lambda_{*}>0$ such that for each $\lambda>\lambda_{*}$ there exists at least one non-zero semi-regular solution $u_{\lambda}$ to problem (2)-(3). Theorem 4 is proven.

The existence of the second (trivial) solution to problem (2)-(3) in Theorem 3 is guaranteed by Condition 2) $(g(x, 0)=0$ for almost each $x \in \Omega)$. We note that the operator $A: X \rightarrow X$ defined by the identity

$$
(A u, v)=\sum_{i, j=1}^{n} \int_{\Omega} a_{i j}(x) u_{x_{i}} v_{x_{j}} d x+\int_{\Omega} c(x) u(x) v(x) d x \quad \forall u, v \in X
$$

in the case of the Dirichlet or Neumann condition, and by the identity

$$
\begin{gathered}
(A u, v)=\sum_{i, j=1}^{n} \int_{\Omega} a_{i j}(x) u_{x_{i}} v_{x_{j}} d x+ \\
\int_{\Omega} c(x) u(x) v(x) d x+\int_{\Gamma} \sigma(s) u(s) v(s) d s \quad \forall u, v \in X
\end{gathered}
$$


in the case of the Robin condition, is self-adjoint, linear, and bounded. The kernel of the operator $A$ coincides with the space $N(L)$. In accordance with Fredholm theory, the negative subspace of the operator $A$ is finite-dimensional and, moreover, if $N(L) \neq\{0\}$, then zero is an isolated point of finite multiplicity in the spectrum of the operator $A$ [16]. The Hilbert space $X$ is densely and compactly (by Condition 2) of Theorem 3) embedded into a reflexive Banach space $\mathbf{L}_{p}(\Omega), p=\frac{q}{q-1}, q>\frac{2 n}{n+2}$ [17]. By analogy with [7] we can show that for the compact mapping $T: \mathbf{L}_{p} \rightarrow \mathbf{L}_{q}$ Conditions 2)-3) of Theorem 1 are fulfilled. Hence, the conditions of Theorem 2 hold true. This is why there exists $\lambda_{*}>0$ such that for each $\lambda>\lambda_{*}$ problem (2)-(3) has at least three solutions. Indeed, applying mountain pass theorem [12, we obtain that for each $\lambda>\lambda_{*}$ there exists also an element $v_{\lambda} \in X$ being a critical point of the functional $J^{\lambda}$ such that $J^{\lambda}\left(v_{\lambda}\right)>0\left(J^{\lambda}\left(v_{\lambda}\right)=\inf _{\gamma \in \Gamma} \sup _{t \in[0,1]} J^{\lambda}(\gamma(t))\right.$, where $\left.\Gamma=\left\{\gamma \in \mathbf{C}([0,1], X): \gamma(0)=0, \gamma(1)=u_{\lambda}\right\}\right)$.

Thus, under the conditions of Theorem 3, the functional $J^{\lambda}$ has at least three different critical points. Therefore, for each $\lambda>\lambda_{*}$ there exist at least two non-trivial solutions to problem (2)(3). The solution $u_{\lambda}$ is semi-regular under the aforementioned assumptions for discontinuities of the nonlinearity [7]. Theorem 3 is proven.

We observe that in work [18] similar theorems on the number of solutions to a one-parametric family of Dirichlet problems for higher order elliptic equations with discontinuous nonlinearities were obtained. The proof of these theorems can be also reduced to the checking the conditions of Theorems 1, 2 of the present work.

\section{BIBLIOGRAPHY}

1. M.A. Krasnoselski. Topological methods in the theory of nonlinear integral equations. GITTL, Moscow. 1956. [Macmillan, New York. 1964.]

2. P.H. Rabinowitz. Some global results for nonlinear eigenvalue problems // J. Funct. Anal. 1971. V. 7, No. 3. P. 487-513.

3. M.A. Krasnoselski. Positive solutions of operator equations. Fizmatgiz, Moscow. 1962. [Noordhoff, Groningen. 1964.]

4. H. Amann. Fixed point equations and nonlinear eigenvalue problems in ordered Banach spases // SIAM Review. 1976. V. 18, No. 4. P. 620-709.

5. P.H. Rabinowitz. Variational methods for nonlinear elliptic eigenvalue problems // Indiana Univ. Math. J. 1974. V. 23, No. 8. P. 729-754.

6. P.H. Rabinowitz. A bifurcation theorem for potentional operators // J. Funct. Anal. 1977. V. 25, No. 4. P. 412-424.

7. V.N. Pavlenko, D.K. Potapov. Existence of a ray of eigenvalues for equations with discontinuous operators // Sibirskij matem. zhur. 2001. V. 42, No. 4. P. 911-919. [Siberian Math. J. 2001. V. 42, No. 4. P. 766-773.]

8. D.K. Potapov. On an existence of a ray of eigenvalues for equations of elliptic type with discontinuous nonlinearities in a critical case // Vestnik St.-Peterb. univer. Ser. 10. Prikl. matem. Inform. Proces. uprav. 2004. No. 4. P. 125-132. (in Russian).

9. D.K. Potapov. Estimation of the bifurcation parameter in spectral problems for equations with discontinuous operators // Ufimskij matem. zhur. 2011. V. 3, No. 1. P. 43-46. [Ufa Math. J. 2011. V. 3, No. 1. P. 42-44.]

10. D.K. Potapov. Estimating of operator norms in eigenvalue problems for equations with discontinuous operators // Izv. Sarat. univer. Novaya ser. Ser. Matem. Mekh. Inform. 2011. V. 11, No. 4. P. 41-45. (in Russian).

11. V.N. Pavlenko. Variational method for equations with discontinuous operators // Vestnik Chelyabinsk. gos. univer. Ser. 3. Matem. Mekh. 1994. No. 1(2). P. 87-95. (in Russian).

12. K.C. Chang. Variational methods for non-differentiable functionals and their applications to partial differential equations // J. Math. Anal. and Appl. 1981. V. 80, No. 1. P. 102-129.

13. F. Riesz, B. Szökefalvi. Leçons d'analyse fonctionnelle. 2ème éd. Akadémiai Kiado, Budapest. 1953. 
14. V.N. Pavlenko, V.V. Vinokur. Resonance boundary value problems for elliptic-type equations with discontinuous nonlinearities // Izv. VUZov. Matem. 2001. No. 5. P. 43-58. [Russ. Math. (Izvestiya VUZ. Matematika). 2001. V. 45, No. 5. P. 40-55.]

15. M.A. Krasnoselski, A.V. Pokrovski. Systems with histeresis. Nauka, Moscow. 1983. [SpringerVerlag, Berlin. 1989.]

16. V.P. Mikhailov. Partial differential equations. Nauka, Moscow. 1983. (in Russian).

17. S.L. Sobolev. Applications of functional analysis in mathematical physics. Nauka, Moscow. 1982. [Translations of Mathematical Monographs, AMS. 1991.]

18. D.K. Potapov. On the number of semiregular solutions in problems with spectral parameter for higher-order equations of elliptic type with discontinuous nonlinearities // Differen. uravn. 2012. V. 48, No. 3. P. 447-449. [Diff. Equat. 2012. V. 48, No. 3. P. 455-457.]

Dmitrij Konstantinovich Potapov, Saint Petersburg State University, Universitetskaya nab., 7/9, 199034, Saint Petersburg, Russia E-mail: potapov@apmath.spbu.ru 